\title{
Frequência alimentar para juvenis de robalo-peva criados em água doce
}

\author{
Feeding frequency for fat snook juvenile in fresh water rearing
}

\section{Camila Fernandes Corrêa ${ }^{[a]}$, Antônio Fernando Gervásio Leonardo ${ }^{[\mathrm{b}]}$, Leonardo Tachibana ${ }^{[\mathrm{c}]}$, Lauzio Corrêa Junior ${ }^{[\mathrm{d}]}$}

[a] Zootecnista, Mestre em Aquicultura, pesquisadora científica do Polo Regional do Vale do Ribeira (APTA, SAA, SP), PariqueraAçu, SP, e-mail: cfcorrea@apta.sp.gov.br.

[b] Biólogo, Doutor em Aquicultura, pesquisador científico do Polo Regional do Vale do Ribeira (APTA, SAA, SP), Pariquera-Açu, SP - Brasil, e-mail: afleonardo@apta.sp.gov.br

[c] Engenheiro Agrônomo, Doutor em Aquicultura, pesquisador científico do Instituto de Pesca (APTA, SAA, SP), São Paulo, SP - Brasil, e-mail: 1tachiba@gmail.com

[d] Tecnólogo em Gestão Ambiental, Pós-Graduando em Análise Ambiental, Faculdades Integradas do Vale do Ribeira (UNISEPE), Registro, SP - Brasil, e-mail: lauziojunior@hotmail.com

\section{Resumo}

Este estudo objetivou verificar qual frequência alimentar é adequada para juvenis de robalo-peva criados em água doce. O delineamento utilizado foi o em blocos casualizados, com três frequências alimentares (uma, duas e quatro vezes ao dia) e quatro repetições, divididas em dois blocos (classes de tamanho de peixes com 0,64 $\pm 0,21 \mathrm{~g}$ e 6,12 $\pm 1,87 \mathrm{~g}$ ). As unidades experimentais foram tanques-rede de $1 \mathrm{~m}^{3}$, estocados com 30 robalos cada, por 60 dias. Ao fim do período experimental os peixes atingiram em média 1,77 $\pm 0,14$ g ou $16,0 \pm 2,08 \mathrm{~g}$, dependendo da classe de peso inicial. Os parâmetros zootécnicos de fator de condição, taxa de crescimento específico, conversão alimentar aparente, coeficiente de variação do comprimento e do peso, e sobrevivência foram submetidos à Análise de Variância e ao teste de Tukey com 5\% de probabilidade. Foram detectadas diferenças apenas para o fator de condição. Ao fim do estudo os robalos alimentados duas vezes ao dia apresentaram maiores valores para o fator de condição, quando comparados aos peixes alimentados uma única vez. As médias para os tratamentos de uma, duas ou quatro alimentações ao dia foram respectivamente $0,870 \pm 0,083 ; 0,946 \pm 0,094$ e 0,909 \pm 0,087. Para a taxa de crescimento específico e conversão alimentar, houve tendência de melhores resultados com o aumento das frequências de alimentação. Concluiu-se, nas condições deste experimento, 
que o fornecimento de ração pelo menos duas vezes ao dia para o robalo-peva, criado em água doce, proporciona um bom desempenho zootécnico e melhor fator de condição.

Palavras-chave: Centropomus parallelus. Aquicultura. Alimentação. Vale do Ribeira.

\begin{abstract}
This study aimed to verify adequate feeding frequency for fat snook juveniles reared in fresh water. The experiment design used was a randomized blocks with three feeding frequencies (one, two and four times per day) and four replications divided in two blocks (fish size class of $0.64 \pm 0.21 \mathrm{~g}$ and $6.12 \pm 1.87 \mathrm{~g}$ ). The experimental units were $1 \mathrm{~m}^{3}$ cages stocked with 30 fat snook each one, during 60 days. At the end of the experimental period the fishes reached averages of $1.77 \pm 0.14 \mathrm{~g}$ or $16.0 \pm 2.08 \mathrm{~g}$ according to initial size class. Growth parameters of condition factor, specific growth rate, apparent food conversion, variation coefficient of length, variation coefficient of weight and survival were submitted to Analyzes Variance and Tukey test with 5\% probability. Differences were detected only for condition factor. At the end of the study, snook fed twice daily presented higher values for condition factor when compared to fish fed only one time. Averages for treatments of one, two or four feedings per day were respectively 0.870 $\pm 0.083 ; 0.946 \pm 0.094$ e $0.909 \pm 0.087$. For specific growth rate and food conversion, there was a tendency of better results and with increase of feeding frequency. It is concluded that, at this experiment conditions, ration feeding at least two times each day for fat snook reared in fresh water allows a good performance and a better condition factor.
\end{abstract}

Keywords: Centropomus parallelus. Aquaculture. Feeding. Vale do Ribeira.

\title{
Introdução
}

O robalo-peva, Centropomus parallelus (Poey 1860), ocorre praticamente em toda a costa brasileira, com distribuição desde a costa da Flórida nos Estados Unidos até Santa Catarina no Brasil (FIGUEIREDO; MENEZES, 1980). No Brasil, o robalo é considerado um peixe nobre e de alto valor comercial, muitas vezes com valor acima do de espécies mundialmente valorizadas, como o salmão e o atum (CEAGESP, 2009).

O robalo-peva pertence à família Centropomidae, sendo carnívoro com preferência por peixes como itens alimentares (TONINI et al., 2007). Outra característica importante é a capacidade desta espécie de se adaptar a ambientes com diferentes salinidades, ou seja, ela é eurihalina, sendo encontrada em águas marinhas, estuarinas e em água doce. Alguns pesquisadores estudaram e comprovaram a versatilidade do robalo em se adaptar a ambientes de diversas salinidades (ROCHA et al., 2005; OSTINI et al., 2007).

Atualmente, as técnicas de reprodução e larvicultura para o robalo-peva já estão estabelecidas e só é viável em laboratórios marinhos. Desde a década de 90, laboratórios brasileiros de piscicultura marinha vêm realizando pesquisas com a finalidade de desenvolver tecnologias de maturação de reprodutores e produção de juvenis (GODINHO et al., 2000; CERQUEIRA; TSUZUKI, 2009).

A respeito do crescimento do robalo-peva, as informações são escassas. Os trabalhos realizados em sua maioria se referem apenas à fase de treinamento alimentar, ou ainda ao crescimento dentro de laboratório em tanques de pequeno volume e em água salgada (OLIVEIRA et al. 2003; SOUZA-FILHO; CERQUEIRA, 2003; CORRÊA; CERQUEIRA, 2007; CORRÊA; CERQUEIRA, 2008; OSTINI et al., 2007; TSUZUKI; BERESTINAS, 2008; CERQUEIRA; TSUZUKI, 2009). Um estudo com o monocultivo do robalo-peva em água doce foi realizado em sistema intensivo, comparando diversas fases de crescimento, e concluiu-se que o robalo teve boa adaptação, mas com médias de pesos inferiores a $100 \mathrm{~g}$ em dois anos de estudos (AMARAL Jr. et al., 2009). Trabalhos em laboratório sobre a adaptação do robalo às diversas salinidades da água demonstram sua versatilidade para criação nos variados ambientes, inclusive em água doce (ROCHA et al., 2005; GRACIA-LÓPEZ et al., 2006; TSUZUKI et al., 2007). Entretanto, alterações fisiológicas podem ocorrer em diferentes salinidades, por isso estudos específicos de criação em água doce, salobra e salgada são importantes.

Rev. Acad., Ciênc. Agrár. Ambient., Curitiba, v. 8, n. 4, p. 429-436, out./dez. 2010 
O aprofundamento dos estudos sobre o crescimento do robalo, em criações marinhas e, principalmente, em água doce, é necessário para definir qual o melhor manejo a ser utilizado para esta espécie, a fim de atingir o tamanho comercial de forma economicamente viável. O estudo do manejo alimentar pode trazer respostas importantes sobre o comportamento alimentar e o crescimento do robalo-peva e, dentro deste, a frequência de alimentação tem grande importância.

Na prática da alimentação devem ser levados em conta a fisiologia e o comportamento de cada espécie de peixe e também o custo investido em ração e mão de obra para este manejo. A frequência alimentar de duas ou mais vezes ao dia é apontada em muitos estudos como a melhor estratégia para um bom desenvolvimento de juvenis de diversas espécies de peixes como, por exemplo, o robalo asiático, Lates calcarifer (SALAMA, 2008); a perca prateada, Bidyanus bidyanus (ROWLAND et al., 2005); o linguado do Atlântico, Hipploglossus hippoglossus (SCHNAITTACHER et al., 2005); o linguado japonês, Paralichtys olivaceus (KIM et al., 2007), e o jundiá, Rhamdia quelen (CARNEIRO; MIKOS, 2005). Por outro lado, Tsuzuki e Berestinas (2008) estudaram as frequências alimentares de uma ou duas vezes ao dia para juvenis de robalo-peva criados em água salgada e não observaram diferenças significativas no desempenho dos peixes após 73 dias de experimento.

Com o objetivo de verificar qual frequência alimentar é adequada para juvenis de robalo-peva criados em água doce, foi realizado um estudo na região do Vale do Ribeira, ao sul do Estado de São Paulo, onde o robalo é encontrado naturalmente no litoral e rios da região.

\section{Materiais e métodos}

O estudo foi realizado na Estação de Piscicultura do Polo Regional do Vale do Ribeira, da Agência Paulista de Tecnologia dos Agronegócios (APTA, SAA, SP), localizada em Pariquera-Açu, SP. Foram utilizados juvenis de robalo-peva, Centropomusparallelus, adaptados à água doce e provenientes do Laboratório de Piscicultura Marinha da Universidade Federal de Santa Catarina (LAPMAR, UFSC), localizado em Florianópolis, SC.

O delineamento experimental usado foi o em blocos casualizados, testando três tratamentos com quatro repetições divididas em dois blocos. Os tratamentos foram as frequências alimentares de uma, duas e quatro vezes ao dia e os blocos foram compostos por duas classes de tamanho inicial: peixes de menor tamanho com 0,64 \pm 0,21 g e 4,33 $\pm 0,42 \mathrm{~cm}$ e peixes de maior tamanho com $6,12 \pm 1,87 \mathrm{~g}$ e $8,26 \pm 0,73 \mathrm{~cm}$.

As unidades experimentais foram 12 tanques-rede de $1 \mathrm{~m}^{3}$, estocados com 30 robalos cada um (19 g ou $183 \mathrm{~g}$ por $\mathrm{m}^{3}$ dependendo do bloco) e instalados em um viveiro de água doce de $600 \mathrm{~m}^{2}$, por um período de 60 dias. A alimentação foi realizada com ração comercial extrusada semidensa (que afunda lentamente) com $45 \%$ de proteína bruta, na taxa diária de $6 \%$ da biomassa, ajustada de acordo com as biometrias realizadas e divididas nas frequências alimentares do experimento. A alimentação de uma vez ao dia foi realizada às $8 \mathrm{~h}$, de duas vezes ao dia às $8 \mathrm{~h}$ e $17 \mathrm{~h}$ e de quatro vezes ao dia às $8 \mathrm{~h}, 11 \mathrm{~h}, 14 \mathrm{~h}$ e $17 \mathrm{~h}$. Todos os peixes foram adaptados à alimentação com a mesma ração comercial na frequência de duas vezes ao dia no mês que antecedeu o experimento.

Semanalmente foram analisados os parâmetros de qualidade de água, que se mantiveram adequados para criação de peixes: temperatura $23,5 \pm 2,4{ }^{\circ} \mathrm{C}$, oxigênio dissolvido $5,21 \pm 1,68 \mathrm{mg} \mathrm{L}^{-1}$, transparência

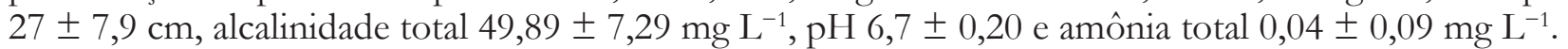

Para avaliação da sobrevivência, crescimento e ajuste da alimentação, foram realizadas três biometrias: inicial, aos 30 dias e aos 60 dias de experimento. Após a anestesia dos peixes com benzocaína (40 mg/L), foram registrados o comprimento total (precisão de $1 \mathrm{~mm}$ ) e o peso (precisão de $0,01 \mathrm{~g}$ ). Os parâmetros zootécnicos de fator de condição (FC), taxa de crescimento específico (TCE), conversão alimentar aparente (CA), coeficiente de variação do comprimento (CV comprimento), coeficiente de variação do peso (CV peso) e sobrevivência, foram submetidos à Análise de Variância e ao teste de Tukey com 5\% de probabilidade $(\mathrm{p}<0,05)$. Anteriormente à análise estatística, os dados em porcentagem passaram pela transformação do arcoseno (SOKAL; ROHLF, 1998). Os dados de comprimento e peso não foram usados diretamente para comparação entre tratamentos, pois foram empregados na divisão dos blocos, tornando as unidades experimentais muito 
diferentes entre si para estes parâmetros. $\mathrm{Na}$ avaliação do crescimento, o peso dos peixes foi utilizado de forma indireta na taxa de crescimento específico, como demonstrado na fórmula. As fórmulas dos parâmetros de desempenho avaliados estatisticamente estão descritas a seguir:

$\mathrm{TCE}=[(\ln$ peso final $-\ln$ peso inicial $) /$ dias $] * 100$

$\mathrm{FC}=\left(\right.$ peso $^{*}$ comprimento $\left.^{-3}\right) * 100$

$\mathrm{CA}=$ peso da ração fornecida/ganho de peso do peixe

$\mathrm{CV}$ comprimento $=$ desvio-padrão do comprimento $/$ média do comprimento

$\mathrm{CV}$ peso $=$ desvio-padrão do peso $/$ média do peso

Sobrevivência $=($ número final de peixes $* 100) /$ número inicial de peixes

\section{Resultados}

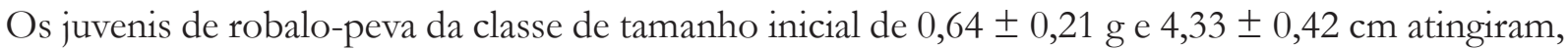
após 60 dias de experimento, peso final de 1,77 \pm 0,14 g e comprimento total de 6,0 $\pm 0,17 \mathrm{~cm}$. Os peixes da classe de tamanho inicial de 6,12 $\pm 1,87 \mathrm{~g}$ e 8,26 $\pm 0,73 \mathrm{~cm}$ atingiram peso final de 16,03 $\pm 2,08 \mathrm{~g}$ e comprimento total de $11,63 \pm 0,50 \mathrm{~cm}$.

Houve diferença estatística entre as frequências de alimentação para o fator de condição dos juvenis (Tabela 1). Aos 60 dias de estudo, os robalos alimentados duas vezes ao dia apresentaram estatisticamente maiores valores para o fator de condição, quando comparados com os peixes alimentados uma vez ao dia. A alimentação de quatro vezes ao dia não diferiu dos demais tratamentos.

Tabela 1 - Fator de condição (FC), taxa de crescimento específico (TCE) e conversão alimentar aparente (CA) de juvenis de robalo-peva, em experimento de 60 dias testando três frequências alimentares

\begin{tabular}{lccc}
\hline Frequência alimentar & FC * & TCE (\%) & CA \\
\hline $1 \times$ ao dia & $0,870 \pm 0,083^{\text {a }}$ & $1,297 \pm 0,166$ & $5,902 \pm 0,975$ \\
$2 \times$ ao dia & $0,946 \pm 0,094^{\mathrm{b}}$ & $1,342 \pm 0,246$ & $5,651 \pm 1,031$ \\
$4 \times$ ao dia & $0,909 \pm 0,087^{\text {ab }}$ & $1,600 \pm 0,240$ & $4,804 \pm 0,982$ \\
\hline
\end{tabular}

Nota: média \pm desvio-padrão; $\mathrm{n}=4$; médias de um mesmo fator com diferentes letras em expoente indicam diferenças significativas $(\mathrm{p}<0,05)$, pela Análise de Variância e teste de Tukey.

Fonte: Dados da pesquisa.

Para a taxa de crescimento específico houve tendência de maior valor numérico para os peixes alimentados quatro vezes ao dia (Tabela 1), mas sem diferenças estatísticas. A conversão alimentar aparente teve valores altos, com menores valores conforme o aumento da frequência alimentar, mas não influenciados estatisticamente pelos tratamentos com média geral de 5,45 $\pm 1,03$.

Também não houve diferenças para o coeficiente de variação do comprimento e para o coeficiente de variação do peso, que tiveram ao fim do experimento respectivamente os valores médios de $0,083 \pm 0,027$ e $0,225 \pm 0,066$, indicando que as frequências de alimentação testadas não influenciaram na homogeneidade de tamanho dos peixes (Tabela 2).

A sobrevivência dos peixes no experimento foi de $98,33 \pm 2,66 \%$. Houve mortalidade de peixes em apenas quatro unidades experimentais, mas sem relação com os tratamentos. 
Tabela 2 - Coeficiente de variação do peso (CV peso), coeficiente de variação do comprimento (CV comprimento) e sobrevivência de juvenis de robalo-peva, em experimento de 60 dias, testando três frequências alimentares

\begin{tabular}{lccl}
\hline Frequência alimentar & CV peso & CV comprimento & Sobrevivência \\
\hline $1 \times$ ao dia & $0,229 \pm 0,080$ & $0,077 \pm 0,026$ & $99,167 \pm 1,667$ \\
$2 \times$ ao dia & $0,216 \pm 0,070$ & $0,096 \pm 0,033$ & $98,333 \pm 3,333$ \\
$4 \times$ ao dia & $0,231 \pm 0,068$ & $0,076 \pm 0,023$ & $97,500 \pm 3,191$ \\
\hline
\end{tabular}

Nota: Média \pm desvio-padrão; $\mathrm{n}=4$.

Fonte: Dados da pesquisa.

\section{Discussão}

Dentro das frequências alimentares testadas de uma, duas ou quatro vezes ao dia para juvenis de robalo-peva criados em água doce, a frequência alimentar recomendada é de duas vezes ao dia, levando em conta os resultados obtidos para o fator de condição no presente estudo. Ao contrário, Tsuzuki e Berestinas (2008) recomendaram a frequência alimentar de uma vez ao dia para juvenis de robalo-peva criados em água salgada, pois não encontraram diferenças de desempenho entre esta frequência e a de duas vezes ao dia. O fator de condição obtido por estes autores ficou entre 1,3 e 1,6, valores maiores do que os obtidos no presente estudo.

A diferença entre os trabalhos devem ter ocorrido principalmente em virtude das diferentes condições ambientais. O estudo de Tsuzuki e Berestinas (2008) foi realizado em um período de 73 dias com juvenis de $4 \mathrm{~g}$ mantidos em água salgada, tanques de pequeno volume em ambiente controlado e utilizando rações extrusadas de baixa ou de alta densidade. O menor valor do fator de condição do atual estudo e altos valores de conversão alimentar obtidos pode ter sido ocasionado pela maior turbidez da água combinada com o uso da ração semidensa que afunda. Esse sistema não permitiu o ajuste diário do alimento fornecido de acordo com a demanda dos peixes, já que não era possível observar a ingestão do alimento.

Como no presente estudo, Tsuzuki e Berestinas (2008) não encontraram influência da frequência de alimentação na conversão alimentar, porém, a conversão foi de 1,5. Valores de conversão altos são comuns para peixes carnívoros, entretanto, o valor de 5,4 obtido no atual experimento deve ser reduzido com estudos específicos de formulação de rações para o robalo-peva. Segundo Guroy et al. (2006), o robalo europeu (Dicentrarchus labrax) alimentado duas ou três vezes ao dia com ração peletizada ou extruzada apresenta crescimento semelhante, no entanto, a eficiência alimentar é melhor com a ração extrusada que flutua.

Um fator que pode ter influenciado a diferença de resultado para as frequências alimentares testadas é o período de jejum de cada tratamento. Para o robalo-peva alimentado duas e quatro vezes ao dia, o intervalo entre alimentações no período noturno foi de 15 horas e no período diurno, de 9 e 3 horas, respectivamente. Para os peixes alimentados apenas uma vez ao dia o período de jejum foi de 24 horas. Booth et al. (2008) observaram para a dourada australiana a taxa de evacuação gástrica de 5 horas para passagem de metade do alimento e de 16 a 20 horas para o total esvaziamento do estomago. Para a tilápia-do-nilo (Oreochromis niloticus), um estudo detectou o retorno ao apetite 5 horas após a alimentação e, também, que peixes alimentados em intervalos iguais ou menores que 3 horas apresentam uma sobrecarga gástrica (RICHE et al., 2004). Essas informações sugerem que os robalos do presente estudo alimentados quatro vezes ao dia tiveram uma sobrecarga diurna de alimento, prejudicando o desempenho. Por outro lado, na alimentação de uma vez ao dia deve ter ocorrido déficit, com um período excessivo de jejum e esvaziamento do trato digestório, o que acarretou piores valores para os parâmetros de crescimento e fator de condição. 
Apesar da tendência de maiores valores, conforme o aumento da frequência alimentar, no presente estudo não houve diferenças estatísticas para a taxa de crescimento específico dos robalos, assim como no estudo de Tsuzuki e Berestinas (2008), que resultaram em taxas de crescimento de $0,9 \%$ a $1,3 \%$, valores semelhantes ao do estudo atual. Para os coeficientes de variação do peso e do comprimento, os resultados obtidos também demonstram que as diferentes frequências alimentares utilizadas propiciaram um crescimento homogêneo dos peixes em cada unidade experimental e também entre cada uma delas. A maioria dos estudos com frequência alimentar não cita os coeficientes de variação, entretanto, este parâmetro é de grande importância na avaliação da qualidade dos peixes produzidos e padronização do produto final.

Especificamente sobre a sobrevivência, os pesquisadores que trabalham com frequência alimentar não relatam efeito dos tratamentos aplicados. O robalo-peva teve uma boa adaptação ao sistema de criação em tanque-rede e água doce, com alta sobrevivência, independente da frequência alimentar utilizada. Alguns estudos indicam alterações fisiológicas para robalos graças às mudanças de salinidade (ROCHA et al., 2005; GRACIA-LÓPEZ et al., 2006; TSUZUKI et al., 2007), entretanto naturalmente esta espécie se adapta a ambientes de salinidades diversas como o mar e rios.

Assim como no presente estudo, muitos experimentos com juvenis de diversas espécies de peixes apontam a frequência alimentar de pelo menos duas vezes ao dia como a mais efetiva para um bom desempenho dos animais, com maior viabilidade técnica e econômica da criação. Em um trabalho de estratégia alimentar para perca prateada, a máxima frequência alimentar para o maior crescimento foi a de duas vezes ao dia, com peixes de $2 \mathrm{~g}$ a $510 \mathrm{~g}$ (ROWLAND et al., 2005). Salama (2008) testou frequências alimentares de duas, três e quatro vezes ao dia e verificou, para juvenis de $59 \mathrm{~g}$ de robalo asiático, que a alimentação em duas refeições diárias resultam em melhor utilização do alimento. Para juvenis de linguado do Atlântico, do linguado japonês e da dourada australiana, frequências de alimentação de duas ou mais vezes ao dia também foram mais adequadas (SCHNAITTACHER et al., 2005; KIM et al., 2007; BOOTH et al., 2008).

Em estudos com juvenis de peixes de água doce nativos do Brasil, as frequências alimentares indicadas variam de acordo com a espécie estudada e a sua fase de desenvolvimento. Carneiro e Mikos (2005) avaliaram o desempenho de juvenis de jundiá de 1,88 g com até quatro alimentações diárias e não encontraram diferenças de desempenho. Por outro lado, Canton et al. (2007), estudando juvenis de 7,47 g da mesma espécie, concluíram que maiores frequências alimentares proporcionaram melhor desempenho, recomendando pelo menos duas alimentações ao dia. Silva et al. (2007) observaram que para o tambaqui (Colossoma macropomum), três alimentações diárias são mais adequadas do que duas alimentações.

No presente estudo, com o robalo-peva criado em água doce, há um indicativo de melhor desempenho utilizando a frequência alimentar de duas vezes ao dia, entretanto, é importante lembrar que existe uma grande influência do sistema de criação e do ambiente no manejo que deve ser utilizado, como se pôde observar nas diferenças dos resultados obtidos por diversos pesquisadores.

\section{Conclusões}

Concluiu-se, para juvenis do robalo-peva, nas condições deste estudo, que a frequência alimentar de pelo menos duas vezes ao dia garante melhor desempenho quando comparado a uma única alimentação diária. O fornecimento de ração duas vezes ao dia garante bom desempenho zootécnico, com melhor valor do fator de condição e diminuição do tempo gasto no manejo alimentar.

\section{Agradecimentos}

À Associação de Mineradores de Areia do Vale do Ribeira e Baixada Santista (AMAVALES) pelo apoio financeiro, aos técnicos da APTA Benedito Martins de Aguiar e Edilberto Rufino de Almeida, pela ajuda nas atividades experimentais, e aos parceiros no fornecimento do material experimental: GUABI, SANSUY e LAPMAR-UFSC.

Rev. Acad., Ciênc. Agrár. Ambient., Curitiba, v. 8, n. 4, p. 429-436, out./dez. 2010 


\section{Referências}

AMARAL Jr., H.; SANTOS, J. J.; GERHARDINGER, R. C. Monocultivo de robalo Centropomus parallelus em água doce. Revista Eletrônica de Veterinária, v. 10, n. 10, 2009. Disponível em: <http://www.veterinaria.org/revistas/redvet/ n101009.html>. Acesso em: 21 jan. 2010.

BOOTH, M. A. et al. Effect of feeding regime and fish size on weight gain, feed intake and gastric evacuation in juvenile Australian snapper Pagrus auratus. Aquaculture, v. 282, p. 104-110, 2008.

CANTON, R. et al. Influência da frequência alimentar no desempenho de juvenis de jundiá. Revista Brasileira de Zootecnia, v. 36, n. 4, p. 749-753, 2007.

CARNEIRO, P. C.; MIKOS, J. D. Frequência alimentar e crescimento de alevinos de jundiá, Rhamdia quelen. Ciência Rural, v. 35, n. 1, p. 187-191, 2005.

CERQUEIRA, V. R.; TSUZUKI, M. Y. A review of spawning induction, larviculture, and juvenile rearing of the fat snook, Centropomus parallelus. Fish Physiol Biochem, v. 35, p. 17-28, 2009.

COMPANHIA DE ENTREPOSTOS E ARMAZÉNS GERAIS DE SÃO PAULO - CEAGESP. Cotações de preços no atacado. Disponível em: <http://www.ceagesp.gov.br/cotacoes>. Acesso em: 11 jan. 2010.

CORREA, C. F.; CERQUEIRA, V. R. Effects of stocking density and size distribution on growth, survival and cannibalism in juvenile fat snook (Centropomus parallelus Poey). Aquaculture Research, v. 38, p. 1627-1634, 2007.

CORREAA, C. F.; CERQUEIRA, V. R. Densidade de estocagem para juvenis de robalo-peva após a larvicultura. Boletim do Instituto de Pesca, v. 34, n. 4, p. 571-576, 2008.

FIGUEIREDO, J. L.; MENEZES, N. A. Manual de peixes marinhos do Sudeste do Brasil III - Teleóstei (2). São Paulo: Museu de Zoologia da USP, 1980.

GODINHO, H. M. et al. Reprodução induzida em robalo Centropomus parallelus Poey, 1860. Brazilian Journal of Veterinary Research and Animal Science, São Paulo, v. 37, n. 1, p. 37-42, 2000.

GRACIA-LÓPEZ, V. et al. Effects of salinity on physiological conditions in juvenile common snook Centropomus undecimalis. Comparative Biochemistry and Physiology, v. 145, part A, p. 340-345, 2006.

GUROY, D. et al. Influence of feeding frequency on feed intake, growth performance and nutrient utilization in European sea bass (Dicentrarchus labrax) fed pelleted or extruded diets. Turkish Journal of Veterinary and Animal Sciences, v. 30, p. 171-177, 2006.

KIM, K-D. et al. Influences of feeding frequency of extruded pellet and moist pellet on growth and body composition of juvenile Japanese flounder Paralichthys olivaceous in suboptimal water temperatures. Fisheries Science, v. 73, p. 745749, 2007.

OLIVEIRA, I. da R. et al. Culture of the fat snook Centropomus parallelus in sea water recirculation system. In: WORLD AQUACULTURE, 2003, Salvador. Anais... Baton Rouge: World Aquaculture Society, 2003. v. 2. p. 534-534.

OSTINI, S. et al. Criação do robalo-peva (Centropomusparallelus) submetido a diferentes densidades de estocagem. Revista Brasileira de Saúde e Produção Animal, v. 8, n. 3, p. 250-257, 2007.

RICHE, M. et al. Effect of feeding frequency on gastric evacuation and the return of appetite in tilapia Oreochromis niloticus (L.). Aquaculture, Amsterdan, v. 234, p. 657-673, 2004.

ROCHA, A. J. S. et al. Metabolic demand and growth of juveniles of Centropomusparallelus as function of salinity. Journal of Experimental Marine Biology and Ecology, v. 316, p. 157-165, 2005.

ROWLAND, S. J. et al. Development of a feeding strategy for silver perch, Bidyanus bidyanus (Mitchell), based on restricted rations. Aquaculture Research, v. 36, p. 1429-1441, 2005. 
SALAMA, A. J. Effects of different feeding frequency on the growth, survival and feed conversion ratio of the Asian sea bass Lates calcarifer juveniles reared under hypersaline seawater of the Red Sea. Aquaculture Research, v. 39, p. 561-567, 2008.

SCHNAITTACHER, G. et al. The effects of feeding frequency on growth of juvenile Atlantic halibut, Hippoglossus hippoglossus L. Aquaculture Research, v. 36, p. 370-377, 2005.

SILVA, C. R. et al. Effect of feeding rate and frequency on tambaqui (Colossoma macropomum) growth, production and feeding costs during the first growth phase in cages. Aquaculture, v. 264, p. 135-139, 2007.

SOKAL, R. R., ROHLF, F. J. Biometry: the principles and practice of statistics in biological research. 3rd ed. New York: W. H. Freeman and Company, 1998.

SOUZA-FILHO, J. J.; CERQUEIRA, V. R. Influência da densidade de estocagem no cultivo de juvenis de robalo-flecha mantidos em laboratório. Pesquisa Agropecuária Brasileira, v. 38, n. 11, p. 1317-1322, 2003.

TONINI, W. C. T. et al. Dieta de juvenis do robalo Centropomus parallelus POEY, 1860 no sul da Bahia, Brasil. Boletim do Instituto de Pesca, v. 33, n. 1, p. 85-91, 2007.

TSUZUKI, M. Y. et al. Salinity tolerance of laboratory reared juveniles of the fat snook Centropomus parallelus. Brazilian Journal of Oceanography, v. 55, n. 1, p. 1-5, 2007.

TSUZUKI, M. Y.; BERESTINAS, A. C. Desempenho de juvenis de robalo-peva Centropomus parallelus com diferentes dietas comerciais e freqüências alimentares. Boletim do Instituto de Pesca, v. 34, n. 4, p. 535-541, 2008.

Recebido: 19/02/2010

Received: 02/19/2010

Aprovado: 12/08/2010

Approved: 08/12/2010 